\title{
MIP models for production lot sizing problems with distribution costs and cargo arrangement
}

\section{Flavio Molina, Reinaldo Morabito \& Silvio Alexandre de Araujo}

To cite this article: Flavio Molina, Reinaldo Morabito \& Silvio Alexandre de Araujo (2016) MIP models for production lot sizing problems with distribution costs and cargo arrangement, Journal of the Operational Research Society, 67:11, 1395-1407, DOI: 10.1057/jors.2016.12

To link to this article: https://doi.org/10.1057/jors.2016.12

\section{Published online: 21 Dec 2017.}

W

Submit your article to this journal ¿

لll Article views: 21

View Crossmark data ¿

Citing articles: 1 View citing articles $\longleftarrow$ 


\title{
MIP models for production lot sizing problems with distribution costs and cargo arrangement
}

\author{
Flavio Molina ${ }^{1}$, Reinaldo Morabito $^{2 *}$ and Silvio Alexandre de Araujo ${ }^{3}$ \\ ${ }^{1}$ Universidade Federal do Triângulo Mineiro, Uberaba, Brazil; ${ }^{2}$ Universidade Federal de São Carlos, São \\ Carlos, Brazil; and ${ }^{3}$ Universidade Estadual Paulista, São José do Rio Preto, Brazil
}

\begin{abstract}
This study presents mixed integer programming (MIP) models for production lot sizing problems with distribution costs using unit load devices such as pallets and containers. Problems that integrate production lot sizing decisions and loading of the products in vehicles (bins) are also modelled, in which constraints such as weight limits, volume restrictions or the value of the cargo loaded in the bins are considered. In general, these problems involve a tradeoff between production, inventory and distribution costs. Lot sizing decisions should take into account production capacity and product demand constraints. Distribution decisions are related to the loading and transport of products in unit load devices. The MIP models are solved by the branch-and-cut method of an optimization package and the results show that these approaches have the potential to address different practical situations.
\end{abstract}

Journal of the Operational Research Society (2016) 67(11), 1395-1407. doi: 10.1057/jors.2016.12; advance published online 27 April 2016

Keywords: lot sizing; distribution costs; cargo unitization; bin packing; integrated problems

\section{Introduction}

The production lot sizing problem can be seen as a problem of optimization of production plans, consisting in defining the quantity of each product or item to be produced in one or more machines in each period of a time horizon, in order to meet the demand for each item and optimize some performance goals. Effective production planning usually involves several factors, such as the production costs involved, the time available for production, the availability of resources and raw materials, the deadlines for meeting the products demands and the setups of the machines when they are relevant to the production process. Each of these factors may have important influences on the total costs and overall performance of the production plan. Reviews of different aspects of lot sizing problems can be found, for example, in Karimi et al (2003), Brahimi et al (2006), Jans and Degraeve (2007, 2008), Robinson et al (2009), Glock et al (2014), Holmbom and Segerstedt (2014) and Beullens (2014).

The costs considered in lot-sizing problems are usually limited to costs of production, inventory, setup and overtime, and other related costs that may be relevant to the production and logistics of the final product are disregarded. For example, in some organizations, end products are packed and placed on pallets to be stored or transported along the supply chain to the customers or distribution centres. The transport cost of products from the factories to the warehouses is directly related to

*Correspondence: Reinaldo Morabito, Production Engineering Department, Universidade Federal de São Carlos, Via Washington Luiz, km.235, 13565905, São Carlos, SP - Brazil.

E-mail: morabito@ufscar.br production lot-sizing decisions. Transport costs may behave differently in relation to lot size, for example, in an economy of scale, that is, high production of the items can make the transport costs per product cheaper. According to Norden and Velde (2005), although transport costs can represent more than $50 \%$ of the logistics cost of a product, in general, they are virtually neglected in modelling lot-sizing problems. Vroblefski et al (2000) highlighted that one of the biggest costs in distribution systems is the cost of transportation and this cost is highly dependent on the volume of the products being transported.

Molina et al (2013) investigated some mathematical models for lot sizing problems with transport costs based on load unitization and presented some preliminary results with these models. This work is an extension of that study where those models are revisited considering decisions about distribution costs and also load arrangement decisions of the products in vehicles, yielding in integrated production lot sizing and product loading (both on pallets and in containers) problems under constraints of limited weight, volume or load value in bins. Several mixed integer programming (MIP) models are presented, which represent these problems and support production planning decisions and the products distribution involved. The models are useful for research and development of more effective solution methods, exploring particular structures, model decomposition, model relaxations, etc. They are also helpful for the performance evaluation of heuristic methods, since they allow (at least for problems of moderate size) an estimation of the optimality gap of heuristic solutions. The performance of these models and the results obtained on solving 
them with the CPLEX optimization package are also discussed and analysed. The results show that these models can be useful for treating various real-life situations.

This paper is presented as follows. Section 2 presents a brief literature review of studies on coupled production planning and distribution decisions. In Section 3, the MIP models are presented that take into account the lot sizing problem with distribution costs using cargo unitization devices such as pallets and containers, as well as a model that integrates lot sizing and loading problems with restrictions of weight, volume or value of cargo loaded in vehicles. In Section 4, an analysis of the computational results obtained with the models in several randomly generated problem instances is performed and Section 5 presents the concluding remarks and some possibilities for future research.

\section{Literature review and background}

To the best of our knowledge, there are only a few studies in the literature that are directly related to the problems studied in this paper, so we first review some papers that have partial connection with this work. Some surveys presented literature reviews on production and distribution planning problems. For example, in Thomas and Griffin (1996) three categories of integrated problems were distinguished: planning the purchase of raw materials and production; production and distribution planning; and also inventory and distribution planning. In Erengüç et al (1999), mathematical models were presented that take into account three different stages of the supply chain. The first looks at suppliers that provide goods or services for various plants, the second is related to the production of finished items to meet a given demand and in the third, the distribution of the final products is taken into consideration. In Rizk and Martel (2001), the authors presented a literature review and a lot sizing model with multiple lines of production and delivery of the demand for customers in the supply chain. A review that considers extensions of the classic lot sizing with transport costs problem appears in Bertazzi and Speranza (1999). Other reviews of integrated lot sizing with distribution problems can be found in Vidal and Goetschalckx (1997) and Sarmiento and Nagi (1999). It is worth noticing that these literature reviews did not present the problem we are dealing with.

More recently, but still not directly related with the problem considered in the present paper, Lee et al (2005) analysed the carrier capacity that is associated with the number of containers (or pallets) needed to load all the production. The items are produced and assigned to containers and the goal is to minimize the number of containers used. The authors proposed a heuristic based on the problem representation as a network flow. Anily and Tzur (2006) considered the demand of the retail stores being met directly from the factory or a warehouse, taking into account the transportation costs. Stecke and Zhao (2007) studied a problem with the make-to-order type of production system. The shipping of the items is outsourced and different options concerning the delivery time are offered. The goal is to plan lot sizes with transportation costs. Melo and Wolsey (2010) presented an alternative formulation for the problem proposed in Stecke and Zhao (2007). In Xiao and Taaffe (2010), models were developed for a problem in which the lot sizing is solved taking into account the costs of production and the delivery rate to final consumers. Other works that treated logistics decisions and lot sizing problems are: Wong (2010) and Seliaman and Ahmad (2009).

Several studies dealt with the lot sizing problem together with decisions on the transport of final products. However, the focus in these articles was not on the loading problem of produced items; rather, it is on other costs involved in the distribution process, such as the routing costs of vehicles that distribute these items (Baumol and Vinod, 1970; Fumero and Vercellis, 1999; Vroblefski et al, 2000; Amorim et al, 2013). There are also some studies that considered the design of integrated lot sizing and transport decision problems between various plants in the same company, that is, they did not take into account transport decisions for customers. Among these articles, we have: Kaminsky and Simchi-Levi (2003), de Matta and Miller (2004), Sambasivan and Schmidt (2002), Sambasivan and Yahya (2005), and Nascimento et al (2010).

More related to the present paper, in Norden and Velde (2005), cargo costs depend on the type of contract established with the logistics operator, that is, they are not only dependent on the volume of items to be transported. The model proposed by the authors was motivated by a practical problem of a European company with monthly fluctuations in the distribution of products between its factory and its warehouses. The company negotiates a long-term contract with the carrier, in which a fixed cost per period is associated with the transport of the items; however, a limited number of pallets or containers are made available at a lower unit cost than the standard cost. The limit to the number of pallets or containers is stipulated based on the forecast demand. If a company needs a larger number of these units in a given period, new units may be used but at higher unit costs. The aim is to minimize the cost of production and distribution of items from the factory to the warehouses. In this problem, transport costs weigh in favour of increasing setups and reducing stocks. This behaviour occurs in this model because transport costs force production of a wider mix of items for better use of pallets or containers. Furthermore, to avoid the use of expensive pallets or containers in a given period, the transport costs force more setups being made and less inventory.

Molina et al (2009) proposed a heuristic method to solve the problem presented in Norden and Velde (2005) and proposed an extension of the model by taking into account the delay in delivery of demand and production capacity constraints. In Molina et al (2013), other extensions of the models originally proposed in Norden and Velde (2005) and Molina et al (2009) representing more general cases, that occur in practice, were analysed and, also, a solution method based on Lagrangian 
relaxation was implemented for one of the proposed extensions. In the present paper, we further extend the models from the literature mainly by considering the combination of the production lot sizing decisions and truck loading decisions without intermediate cargo unitization devices such as pallets and containers. We have not found any paper in the literature that considers such combination.

The main contributions of the present paper are: the proposition of several innovative MIP models considering decisions about distribution costs and also load arrangement decisions of the products in vehicles, yielding in integrated production lot sizing and product loading (both on pallets and in containers) problems under constraints of limited weight, volume or load value in bins; the performance analysis of these models obtained on solving them with the CPLEX optimization package. The results show that these approaches are able to address different practical situations.

\section{MIP models}

This section presents three MIP models for lot sizing problems with transport costs, where the goods are transported using unit load devices such as pallets or containers, as well as a model for an integrated MIP problem by combining production lot sizing and trucks loading decisions. The first model considers the case wherein is required to load the items onto pallets without mixing items, that is, each pallet can be loaded only with a single type of item, which is usual in most production processes, especially in manufacturing environments, such as soft drinks companies, canned food warehouse, spinning industries, among others. The transport costs are directly related to the number of pallets required to transport the items.

The second and third models extend the first one by considering that, after allocating the items on pallets, the latter are loaded on trucks of a homogeneous fleet (second model) and heterogeneous fleet (third model), and the trucks go to the same destination (ie a warehouse). In practice, the second and third models appear most commonly in companies with high production, unlike the first case, in which the amount produced is not always large enough to request the dedication of a truck. Observe that in these cases the transport costs are directly related to the number of trucks required to transport the pallets. The fourth model represents the practical situation where the produced items are loaded directly onto trucks, without intermediate cargo unitization devices. This case occurs, for example, when items are large enough to be loaded on pallets or other unitization devices, as in some furniture companies.

\subsection{Model 1-Loading onto pallets without mixing items}

Unlike the models reviewed in the literature, for example in Norden and Velde (2005), the first MIP model considers production of items that have different sizes and cannot be mixed on the same pallet (Figure 1). Therefore, it is necessary to

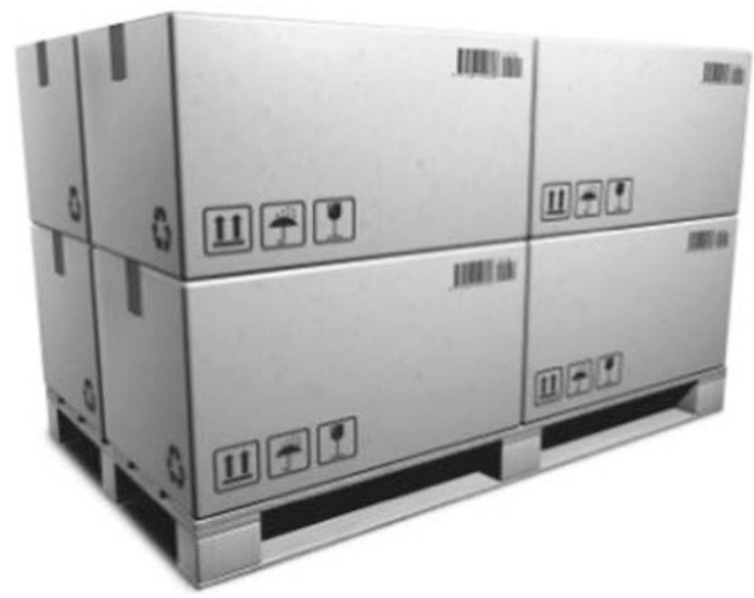

Figure 1 Pallet loading (authorship: http://www.freedigitalphotos. net).

define the capacity of the pallet for each type of item. These data can be obtained in advance by solving the so-called Manufacturer's Pallet Loading Problem (eg Alvarez-Valdes et al, 2005; Morabito and Pureza, 2009; Birgin et al, 2010) as a pre-processing step for each type of item. This loading problem assumes that the items are arranged in horizontal layers on the surface of the pallet, which is a usual practice for producers to facilitate the arrangement and load stability. The parameters and variables of the model are described below.

\section{Parameters:}

$i=1,2, \ldots, n \quad$ Number of types of items.

$t=1,2, \ldots, T \quad$ Number of periods in the planning horizon.

$s_{i t}$ Setup cost for the production of item $i$ in period $t$.

$h_{i t}^{+} \quad$ Unit inventory cost of item $i$ in period $t$.

$h_{i t}^{-} \quad$ Penalty for delay of one unit of item $i$ in period $t$.

$d_{i t} \quad$ Demand for item $i$ in period $t$.

$b_{i} \quad$ Time required to produce a unit of item $i$.

$q_{i} \quad$ Setup time for the production of item $i$.

$\mathrm{Cap}_{t} \quad$ Production capacity in period $t$.

$M \quad$ A sufficiently large positive number.

$P_{i} \quad$ Number of items of type $i$ that can be placed on the same pallet (data obtained beforehand by solving a Pallet Loading Problem for each item $i$ ).

$c_{0} \quad$ Fixed monthly cost of the contract.

$c_{1} \quad$ Unit transport cost of first $R$ pallets used.

$c_{2} \quad$ Unit transport cost of the other pallets used $\left(c_{2}>c_{1}\right)$.

$R \quad$ The contracted number of hired pallets with cheaper cost $c_{1}$.

Decision variables:

$X_{i t}$

Amount to be produced of item $i$ in period $t$. 


$\begin{array}{ll}I_{i t}^{+} & \text {Inventory of item } i \text { in period } t . \\ I_{i t}^{-} & \text {Backlog of item } i \text { in period } t . \\ Y_{i t} & \text { Binary variable indicating the production of } \\ & \text { item } i \text { in period } t\left(Y_{i t}=1 \text { if } X_{i t}>0 ; Y_{i t}=0\right. \\ & \text { otherwise). } \\ A_{i t} & \text { Number of pallets transported containing item } \\ & i \text { in period } t \text { with unit cost } c_{1} . \\ B_{i t} & \text { Number of pallets transported containing item } \\ & i \text { in period } t \text { with unit } \operatorname{cost} c_{2} .\end{array}$

The transport costs are piecewise linear and convex in relation to the number of pallets used. Note that from the quantity $R$, the transportation cost per unit changes from $c_{1}$ to $c_{2}$. Model 1 is defined by:

Model 1:

$$
\begin{aligned}
z= & \min \sum_{i=1}^{n} \sum_{t=1}^{T}\left(h_{i t}^{+} I_{i t}^{+}+h_{i t}^{-} I_{i t}^{-}+s_{i t} Y_{i t}\right) \\
& +\sum_{t=1}^{T}\left(c_{0}+c_{1} \sum_{i=1}^{n} A_{i t}+c_{2} \sum_{i=1}^{n} B_{i t}\right)
\end{aligned}
$$

Subject to:

$$
\begin{gathered}
I_{i, t-1}^{+}-I_{i, t-1}^{-}+X_{i t}-I_{i, t}^{+}+I_{i, t}^{-}=d_{i t} \\
i=1, \ldots, n ; t=1, \ldots, T \\
X_{i t}-M Y_{i t} \leqslant 0 \quad i=1, \ldots, n ; t=1, \ldots, T \\
\sum_{i=1}^{n}\left(b_{i} X_{i t}+q_{i} Y_{i t}\right) \leqslant C a p_{t} \quad t=1, \ldots, T \\
B_{i t}+A_{i t} \geqslant \frac{X_{i t}}{P_{i}} \quad i=1, \ldots, n ; t=1, \ldots, T \\
0 \leqslant \sum_{i=1}^{n} A_{i t} \leqslant R \\
t=1, \ldots, T \\
I_{i 0}^{+}=I_{i 0}^{-}=I_{i T}^{-}=I_{i T}^{-}=0, \quad i=1, \ldots, n \\
X_{i t} \geqslant 0, I_{i t}^{+} \geqslant 0, I_{i t}^{-} \geqslant 0 \\
i=1, \ldots, n ; t=1, \ldots, T \\
A_{i t}, B_{i t} \in Z^{+}, Y_{i t} \in\{0,1\} \\
i=1, \ldots, n ; t=1, \ldots, T
\end{gathered}
$$

The objective function (1) minimizes the total cost given by the sum of the production costs (inventory, backlog and machine setup) with the transportation costs (contract cost, cost of cheaper pallets and cost of more expensive pallets).
Constraints (2) refers to the balancing constraints of production, demand, inventory and backlog of items. Constraints (3) ensure that there is production only when the setup cost and time have been considered. Constraints (4) are restrictions on production capacity. Constraints (5) ensure that a sufficient number of pallets to transport each item type is used. Constraints (6) limit the use of pallets at a lower cost to $R$. Constraints (7) impose that initial and final inventory and backlog levels are equal to zero and constraints (8) ensure the non-negativity of production, inventory and backlog variables. Finally, in (9), the domains of binary and integer variables are defined. For this model, $M$ is set as the sum of the demands of all items throughout the planning horizon, $M=\sum_{i=1}^{n} \sum_{t=1}^{T} d_{i t}$.

To get a sense of the model dimension, it can easily be shown that the model has $(3 n T)$ real variables, $(2 n T)$ integer variables and $(n T)$ binary variables. Furthermore, the model has $T$ $(3 n+2)+4 n$ constraints. Thus, for an example with 12 periods and 20 types of items, the model has 720 real variables, 480 integer variables, 240 binary variables and 824 constraints.

3.1.1. Example problem: The following is a simplified example to illustrate the importance of the solution of the integrated lot sizing problem with transportation costs. Consider the following values for a problem with $n=5$ items and $T=5$ periods. The inventory cost per item and per period is $h_{i t}^{+}=3 \forall i, t$, the cost of delay per item and per period is $h_{i t}^{-}=$ $30 \forall i, t$ and the machine setup cost for the production of items is $s_{i t}=100 \forall i, t$. The production time of each item is $b_{i}=1 \forall i$. The machine setup time for production of items is equal to $q=(17,17,12,10,17)$. The capacity in each period is Cap $_{t}=$ $316 \forall t$. The pallet loading capacity for each item is $P=(56,101,87,124,89)$. The unit cost of the cheaper pallets is $c_{1}=50$ and of the more expensive pallets cost is $c_{2}=200$. The maximum number of cheaper pallets that can be used for each period is $R=3$. The demand for the items is given by the following matrix, the rows being the items and the columns being the periods.

$$
d=\left(\begin{array}{lllll}
7 & 11 & 8 & 13 & 11 \\
58 & 94 & 79 & 108 & 64 \\
39 & 46 & 85 & 33 & 32 \\
61 & 75 & 73 & 51 & 47 \\
33 & 62 & 41 & 50 & 34
\end{array}\right)
$$

Solving this problem disregarding the transport of items, that is, first solving the lot sizing problem, regardless of transportation decisions, and then with the solution of the lot sizing problem determining the number of pallets and the transport costs, we obtain a solution with a production cost equal to 2793 . To transport the quantities produced, five pallets are needed in the first period, four pallets in the second and third periods, and five pallets in the fourth and fifth periods, resulting in a shipping 
cost of 2350. Thus, the total cost of production and transportation is 5143 .

On the other hand, by solving the problem using Model 1, we obtain an optimal solution with a total cost of 4907, with production costs equal to 3157 and shipping costs equal to 1750. That is, a total cost lower than that calculated separately in the previous paragraph. With this small example, we can see that solving the problems of production and transport separately and sequentially, as is usually done in practice, may not provide the best solution for the integrated problem.

\subsection{Model 2-Transport of pallets on homogeneous fleet}

The second MIP model for the lot sizing problem with transport costs considers that the items to be produced have different types and sizes and cannot be mixed on the same pallet, in the same way as Model 1. However, in addition, the model assumes that, after allocating the items on pallets, the latter are loaded on trucks of a homogeneous fleet (Figure 2).

In this case, transport costs are not directly related to the number of pallets used, but to the number of trucks required to transport the pallets. In addition to the problem of loading items (equal-sized) on pallets (one problem for each item type), there is the problem of loading pallets (of equal size) in the truck. Both packing problems can be considered in a pre-processing, solving the Manufacturer's Pallet Loading Problem and assuming that the pallets are arranged in horizontal layers within the truck bed, which is also usual in practice. With this preprocessing, the maximum number of pallets that can be transported on a truck is calculated. Thus, besides the number of items of type $i$ that are loaded on each pallet $\left(P_{i}\right)$, it is now also necessary to previously calculate the number of pallets, assuming equal dimensions (length and width), can be loaded on the truck $\left(P^{\prime}\right)$ (usually, no more than two layers of each pallet on the truck due to its height limitation).

\section{Additional parameters of Model 2:}

$\begin{array}{ll}P^{\prime} & \text { Maximum number of pallets that can be } \\ \text { transported on a truck. } & \\ c_{1} & \text { Unit cost of the first } R^{\prime} \text { used trucks. }\end{array}$

$\begin{array}{ll}c_{2} & \text { Unit cost of other trucks }\left(c_{2}>c_{1}\right) . \\ R^{\prime} & \text { Contracted number of hired trucks with } \\ & \left.\text { cheaper cost } c_{1} \text { (similarly to } R \text { of Model } 1\right) .\end{array}$

Additional decision variables:

$A_{t} \quad$ The number of trucks used in period $t$ with unit cost $c_{1}$.

$B_{t} \quad$ The number of trucks used in period $t$ with unit $\operatorname{cost} c_{2}$.

$Z_{i t} \quad$ Number of pallets required to transport the items of type $i$ produced in period $t$.

Model 2 is given by:

Model 2:

$$
\begin{aligned}
z= & \min \sum_{i=1}^{n} \sum_{t=1}^{T}\left(h_{i t}^{+} I_{i t}^{+}+h_{i t}^{-} I_{i t}^{-}+s_{i t} Y_{i t}\right) \\
& +\sum_{t=1}^{T}\left(c_{0}+c_{1} A_{t}+c_{2} B_{t}\right)
\end{aligned}
$$

Subject to :

$$
\begin{gathered}
(2),(3),(4),(7),(8) \\
B_{t}+A_{t} \geqslant \frac{1}{P^{\prime}} \sum_{i=1}^{n} Z_{i t} \quad t=1, \ldots, T \\
Z_{i t} \geqslant \frac{X_{i t}}{P_{i}} \quad i=1, \ldots, n ; t=1, \ldots, T \\
0 \leqslant A_{t} \leqslant R^{\prime} \quad t=1, \ldots, T \\
A_{t}, B_{t}, Z_{i t} \in Z^{+}, Y_{i t} \in\{0,1\} \\
i=1, \ldots, n ; t=1, \ldots, T
\end{gathered}
$$

The objective function (10) and constraints (13) and (14) were simply adapted from (1), (6) and (9), respectively, for the

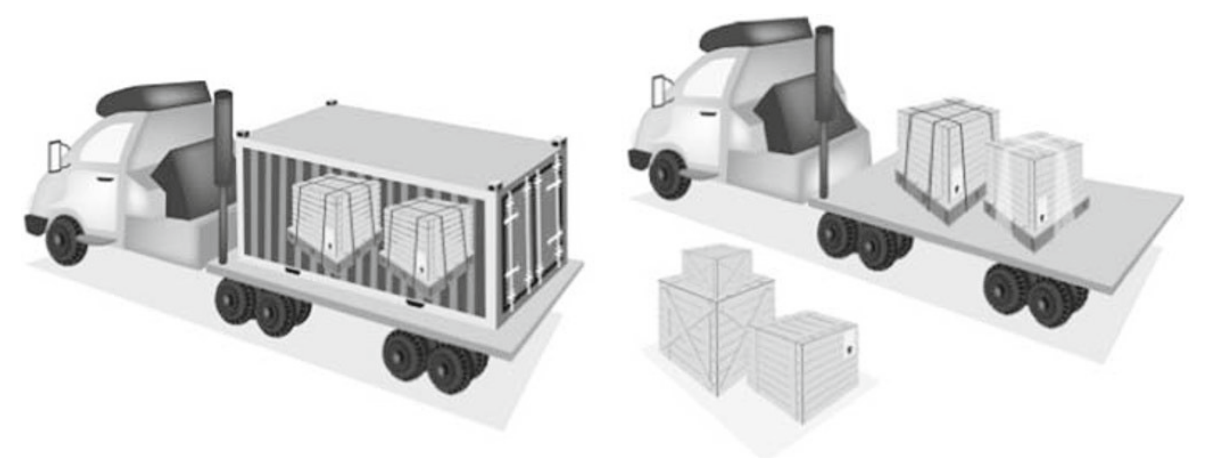

Figure 2 Trucks of a homogeneous fleet (authorship: http://www.freedigitalphotos.net). 
problem on hand. Constraints (11) determine the number of trucks required to transport the pallets at the lower cost and at the more expensive cost. Constraints (12) refer to the number of pallets required to transport each item type in each period. As the items are transported to the same destination (ie a warehouse) and the pallets are considered to be of equal dimensions, there is no need for transport variables with information on which items are being carried by each truck.

Model 2 has $2 T(n+1)$ integer variables while the remaining variables keep the same numbers as Model 1. Furthermore, Model 2 has $T(3 n+3)+4 n$ constraints. For example, for a problem with 20 types of item and 12 time periods, the model has 720 real variables, 264 integer variables (216 less than Model 1), 240 binary variables and 836 constraints.

\subsection{Model 3-Transport of pallets on heterogeneous fleet}

The third MIP model for the lot sizing problem with transportation costs extends the models of Sections 3.1 and 3.2. It includes the existence of various types of trucks with different capacities and costs, which occurs in practice in heterogeneous fleet situations. In the same way as for the cases in Sections 3.1 and 3.2, the packing problem for loading pallets (of equal size) in each truck can be resolved by pre-processing, solving the Manufacturer's Pallet Loading Problem (a different problem for each type of truck).

Note that the following model involves deciding how many trucks of each type to use, which differs from previous models in that it provides only a number, whether pallet or trucks, without the need to choose between different options of trucks with different costs. Therefore, the decision is now related to the cost of each truck. Smaller trucks have lower unit cost, but have restricted capacity for fewer pallets, not necessarily proportional to the reduction in cost. For example, a truck can transport half of the volume of another truck and it does not necessarily cost half the price. Consider the following data and variables in addition to those from Models 1 and 2:

Additional parameters of Model 3:

$k=1, \ldots, K$
$P_{k}^{\prime}$
$c_{1 k}$
$c_{2 k}$
$R_{k}^{\prime}$

Number of different types of trucks. Maximum number of pallets that can be carried in the truck of type $k$. Unit cost of the first $R_{k}^{\prime}$ trucks of type $k$ used. Unit cost of additional trucks of type $k$ $\left(c_{2 k}>c_{1 k}\right)$.

Contracted number of trucks of type $k$ with cheaper cost $c_{1 k}$ (similarly to $R$ and $R^{\prime}$ of Models 1 and 2).

Additional decision variables:

$A_{k t} \quad$ Number of trucks of type $k$ used in period $t$ with unit $\operatorname{cost} c_{1 k}$.
$B_{k t}$

Number of trucks of type $k$ used in period $t$ with unit cost $c_{2 k}$.

$Z_{k t}^{\prime} \quad$ Number of trucks of type $k$ to transport pallets in period $t$.

Model 3 is given by:

Model 3:

$$
\begin{aligned}
z= & \min \sum_{i=1}^{n} \sum_{t=1}^{T}\left(h_{i t}^{+} I_{i t}^{+}+h_{i t}^{-} I_{i t}^{-}+s_{i t} Y_{i t}\right) \\
& +\sum_{t=1}^{T}\left(c_{0}+\sum_{k=1}^{K}\left(c_{1 k} A_{k t}+c_{2 k} B_{k t}\right)\right)
\end{aligned}
$$

Subject to :

$$
\begin{gathered}
(2),(3),(4),(7),(8),(12) \\
A_{k t}+B_{k t} \geqslant \frac{Z_{k t}^{\prime}}{P_{k}} \quad t=1, \ldots, T ; k=1, \ldots, K \\
\sum_{k=1}^{K} Z_{k t}^{\prime}=\sum_{i=1}^{n} Z_{i t} \quad t=1, \ldots, T \\
0 \leqslant A_{k t} \leqslant R_{k}^{\prime} \quad t=1, \ldots, T ; k=1, \ldots, K \\
A_{k t}, B_{k t}, Z_{i t}, Z_{k t}^{\prime} \in Z^{+}, Y_{i t} \in\{0,1\} \\
i=1, \ldots, n ; t=1, \ldots, T ; k=1, \ldots, K
\end{gathered}
$$

The objective function (15) has been modified with respect to (1) to depict the new transport costs. Constraints (16) calculate the number of trucks needed to transport the pallets in period $t$. Constraints (17) ensure that the number of pallets transported by trucks is equal to the number of pallets required to convey the production of each period. Note that the model does not have any information on which item is being loaded onto what type of truck, but this information is not relevant for the solution of the problem because we are considering that the pallets have the same size and the items have the same destination (ie the warehouse). Constraints (18) limit the use of type $k$ trucks with lower $\operatorname{cost} R_{k}^{\prime}$.

Model 3 has $(n T+3 n K)$ integer variables and the same number of real and binary variables as the other models. Regarding the number of constraints, Model 3 has $(3 n T+2 T+$ $4 n+2 K T)$ constraints. For example, for a problem with 12 periods and 20 different kinds of items and 2 types of trucks, the model has 720 real variables, 312 integer variables, 240 binary variables and 872 constraints.

Model 3 can be modified for the specific case in which there is only one kind of contract cost $\left(c_{l k}\right)$ for each type of truck $k$. In this case, the variables $B_{k t}$ are not considered and the decisions only involve the choice of each type of truck, which, in turn, are only available in limited quantities $\left(R_{k}^{\prime}\right)$. The problem can become unsolvable if the number of trucks 

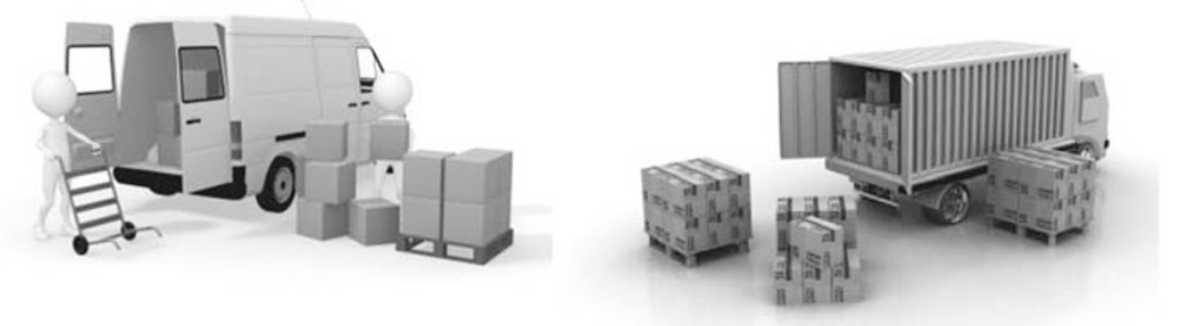

Figure 3 Transportation directly in trucks - without pallets (authorship: http://www.freedigitalphotos.net).

is not sufficient to carry the entire production. This change can be seen as an adaptation of Model 3 in which the cost $c_{2 k}$ is artificially set to a sufficiently high value. For the computational tests presented in the next section, we used the modified version of Model 3.

\subsection{Model 4-Integrated model for lot sizing and bin packing}

The following is a model for the integrated MIP problem by combining the production lot sizing decisions and truck loading decisions without intermediate cargo unitization devices such as pallets and containers (Figure 3). Note that with this condition, unlike previous models, the item packing parameters cannot be calculated a priori, because the packing of an item depends directly on the sizes and quantities of other items that are being produced in the same period. It is therefore necessary to develop models that integrate the lot sizing problem with the packing problem in a monolithic and simultaneous way, such that the decisions of the two problems are interdependent.

Due to the difficulty of developing and solving integrated lot sizing models with the packing problem taking into account the three dimensions involved in the physical arrangement (lengths, widths and heights of the items and vehicles), this study considers only one dimension for packing problem. In this case, one can think that this dimension is the weight, volume or value of the goods. The cargo value constraint may happen with some carriers on the basis of the insurance value limits imposed when insuring the load, which is common in some countries, such as Brazil. It can be seen that the proposed one-dimensional packing model is based on the Kantorovich's (1960) model, adapted to represent the fact that loading is done in every period.

Model 4 parameters:

$w_{i} \quad$ Length (or weight or volume or value) of the

$W \quad$ Capacity of each truck given in length (or item type $i$. weight or volume or value limit).

M Sufficiently large positive number.

$c \quad$ Unit cost per truck used for the transport of produced items.
Decision variables:

$Z_{i k t} \quad$ Number of items of type $i$ allocated to truck $k$ in period $t$.

$A_{k t} \quad$ Binary variable indicating whether truck $k$ is used in period $t$.

In Models 1, 2 and 3, the transport variables are integer (instead of binary) and indicated how many trucks are used. In this model, the carrier is a binary variable indicating whether the truck $k$ is used or not in each period $t$. Moreover, the cost of transport $(c)$ is considered simply as linear (rather than piecewise linear convex). However, the model is still valid for the convex case and, in this case, it simply replaces the constant $c$ for a cost vector, where $R$ first trucks have value $c_{1}$ and the others are of value $c_{2}$. In addition, to consider piecewise linear concave costs, one need to simply add a constraint ordering the variables $A_{k t}$ to ensure that truck $k+1$ will only be used if truck $k$ is already in use.

Model 4:

$$
z=\min \sum_{i=1}^{n} \sum_{t=1}^{T}\left(h_{i t}^{+} I_{i t}^{+}+h_{i t}^{-} I_{i t}^{+}+s_{i t} Y_{i t}\right)+\sum_{t=1}^{T} \sum_{k=1}^{M} c A_{k t} .
$$

Subject to :

$$
\begin{array}{r}
I_{i(t-1)}^{+}-I_{i(t-1)}^{-}+X_{i t}-I_{i t}^{+}+I_{i t}^{-}=d_{i t} \\
t=1, \ldots, T ; i=1, \ldots, n \\
\sum_{i=1}^{n}\left(b_{i} X_{i t}+q_{i} Y_{i t}\right) \leqslant C a p_{t} \quad t=1, \ldots, T \\
X_{i t} \leqslant \sum_{j=1}^{T} d_{i j} Y_{i t} \quad t=1, \ldots, T ; i=1, \ldots, n \\
\sum_{i=1}^{n} w_{i} Z_{i k t} \leqslant W A_{k t} \quad t=1, \ldots, T ; k=1, \ldots, M \\
\sum_{k=1}^{M} Z_{i k t}=X_{i t} \quad t=1, \ldots, T ; i=1, \ldots, n
\end{array}
$$




$$
\begin{aligned}
& X_{i t}, I_{i t}^{+}, I_{i t}^{-} \geqslant 0 ; Z_{i k t} \in \mathbb{N} \quad t=1, \ldots, T ; i=1, \ldots, n ; \\
& k=1, \ldots, M \\
& \quad Y_{i t}, A_{k t} \in\{0,1\} t=1, \ldots, T ; i=1, \ldots, n ; k=1, \ldots, M
\end{aligned}
$$

Note that, unlike Models 1, 2 and 3, Model 4 contains information on loading the items in each truck. This is due to the variables $Z_{i k t}$, obtained from the packing problem. Remember that Models 1, 2 and 3 contain, due to preprocessing, only the maximum number of pallets that can be loaded in a truck. Constraints (21), (22) and (23) are related to the lot sizing problem and Constraints (24) and (25) to the packing of end products. Constraints (25) are the coupling constraints to integrate the lot sizing and packing decisions, as they relate the production variables to the loading variables. The model consists of determining the lot sizes and the number of trucks in order to minimize the sum of the costs of production and transport (20). Note that Constraints (24) may represent weight limit or volume limit or maximum value. Optionally, we could have more than one of these limits in Constraints (24) representing these maximum values and, thus, limiting the model simultaneously.

Model 4 contains $n T(M+1)$ integer variables, $T(n+M)$ binary variables and $T(3 n+M+1)$ restrictions. Note that there is a need to set the value of $M$, because if it is too large, the number of variables and constraints will also be large. On the other hand, if this number is too small, it may be that the problem becomes infeasible. In this work, the number of available trucks $(M)$ is determined by multiplying the lower bound on the number of bins required to transport the total demand. In other words, the number of available trucks is defined by the equation with $M=$ $\left\lceil\beta \sum_{i=1}^{n} \sum_{t=1}^{T} w_{i} d_{i t} / W\right\rceil$ with $\beta>1$. It should be noted that the value of $M$ obtained in this way is much greater than the number of trucks required to transport the production because, for each period, the problem will have $M$ trucks available. In practice, the value of $M$ is set by the company (as a parameter of the problem), being equal to the number of trucks available in each period. If the fleet is considered unlimited, that is, there is no upper limit on the number of trucks that can be used in each period, Model 4 remains valid but it is then necessary to obtain an upper bound on the number of trucks.

It should be mentioned that, for the specific case of the integrated model where there is only one production period and without any capacity constraints, the integrated lot sizing problem with one-dimensional packing could be just seen as a one-dimensional packing problem. According to Coffman et al (1997), this problem is classified as NP-complete and, thus, this current integrated problem is also NP-complete.

\section{Computational analysis of the models}

In this section, a computational analysis of the models is performed initially for Models 1, 2 and 3 that consider distribution costs and then for Model 4, which takes into account the integrated problem of lot sizing and loading arrangements. The models were coded in AMPL modelling language and solved by the CPLEX 11.1.1 computer package with default parameters, using a Pentium Core 2 Duo $2 \mathrm{GHZ}$ with $2 \mathrm{G}$ of RAM and the Windows XP operating system.

The data used were originally inspired in a single real-life data set that a European manufacturing company made available by Norden and Velde (2005). This manufacturing company had heavily fluctuating monthly shipments between its manufacturing plant and its central warehouse, and had negotiated a transportation capacity reservation contract with a logistics service provider. This contract allowed the shipper to use, in each period, any desired portion of a reservation fixed transportation capacity in exchange for a guaranteed price, which was lower than the expected price on the spot market. The logistics service provider also had some benefits on such type of contract, which included the establishment of a longterm business relationship and the reduction on the need to find new customers. Based on this single data set from this company, problem instances were randomly generated in order to reflect other characteristics of practical settings. Basically, we varied the number of items and periods, representing different product portfolios and different planning horizons, and the available capacities and the transportation costs, representing different situations imposed by the market.

\subsection{Computational analysis of the lot sizing models with distribution costs}

For the classes of Model 1, the following data were used, based on Norden and Velde (2005) and Molina et al (2009). Table 1 shows two kinds of fixed data, constant values and ranges. When the values are represented by an interval, this means that the given value was chosen randomly and uniformly within this range. In the process of generating the data to simulate the seasonality of demand, a significant increase in demand was made every five periods, for this, the demand $d_{i t}$ was multiplied by 1.5 . The production capacity in each period was generated in a way that it depended on setup and production times and the

Table 1 Fixed data for the models

\begin{tabular}{cc}
\hline$s_{i t}$ & 50 \\
$h_{i t}^{+}$ & 3 \\
$h_{i t}^{-}$ & 10 \\
$b_{i}$ & 1 \\
$q_{i}$ & {$[10,30]$} \\
$E D_{i}$ & {$[40,700]$} \\
$d_{i t}$ & {$\left[\left\lfloor\frac{E D_{i}}{2}\right\rfloor,\left\lceil\frac{3 E D_{i}}{2}\right\rceil\right]$} \\
$P_{i}$ & {$[50,150]$} \\
$F R$ & $1 / 4$ \\
\hline
\end{tabular}


demand for each item, using the following formula:

$$
\operatorname{Cap}_{t}=\left\lfloor\frac{\sum_{i=1}^{n} \sum_{j=1}^{T}\left(b_{i} d_{i j}+q_{i}\right)}{\alpha T}\right\rfloor \quad t=1, \ldots, T
$$

where $\alpha$ is a parameter to control the slack in capacity. The maximum number of inexpensive pallets, in other words the value $R$, was calculated as the fraction of average demand which is transported to the $\cos t c_{1}$. The value of $F R$ means that $R$ should be generated so that for a fraction $F R$ of the periods (for example, $F R=1 / 4$ ), the demand must be below $R\left(\sum_{i=1}^{n} P_{i} / n\right)$. In this adaptation value for Model 2, we considered the number of pallets that can be carried in the truck $\left(P^{\prime}\right)$, that is to say, $R^{\prime}$ must be generated so that for a fraction $F R=1 / 4$ of the periods, demand should be less than $\left.\left.R^{\prime}\left[\left(\sum_{i=1}^{n} P_{i}\right) / n\right) / P^{\prime}\right)\right]$. As Model 3 supposes different sizes of trucks and smaller trucks are limited to $R$, in this case $F R$ is interpreted as the fraction of periods when the demand is below $R^{\prime}\left[\left(\sum_{i=1}^{n} P_{i}\right) / P_{k}^{\prime}\right]$.

In addition to these data, there were four other data defining the classes for specific data instances: number of items $(n)$, number of periods $(T)$, transportation costs $\left(c_{1}\right.$ and $\left.c_{2}\right)$ and the slackness control in capacity $(\alpha)$. Sixteen classes of problems were generated (classes $1,2, \ldots, 16$ ) with numbers of items equal to 50 and 150, and numbers of periods equal to 20 and 50. For each of the sixteen classes, 50 instances were generated, which differ only by data that were generated randomly within the predetermined ranges. Table 2 shows the dimensions of the classes of instances 1-16. It is worth noticing that the periods are all of equal length and represent typically months.

In addition to the data generated for Model 1, in Model 2, $P^{\prime}$ was generated uniformly within the range $[40,80]$ (ie, $P^{\prime} \in$ $[40,80])$. The rest of the data was kept the same as the data for Model 1. Note that, now, the costs are for the number of trucks. In Model 3, as mentioned earlier, a special adaptation of Model 1 was considered, where the costs for the use of extra

Table 2 Variable data for model 1

\begin{tabular}{lccccc}
\hline & \#Item $(n)$ & \#Period $(T)$ & $\alpha$ & $c_{1}$ & $c_{2}$ \\
\hline Class 1 & 50 & 20 & 0.85 & 50 & 200 \\
Class 2 & 50 & 50 & 0.85 & 50 & 200 \\
Class 3 & 150 & 20 & 0.85 & 50 & 200 \\
Class 4 & 150 & 50 & 0.85 & 50 & 200 \\
Class 5 & 50 & 20 & 1.00 & 50 & 200 \\
Class 6 & 50 & 50 & 1.00 & 50 & 200 \\
Class 7 & 150 & 20 & 1.00 & 50 & 200 \\
Class 8 & 150 & 50 & 1.00 & 50 & 200 \\
Class 9 & 50 & 20 & 0.85 & 150 & 500 \\
Class 10 & 50 & 50 & 0.85 & 150 & 500 \\
Class 11 & 150 & 20 & 0.85 & 150 & 500 \\
Class 12 & 150 & 50 & 0.85 & 150 & 500 \\
Class 13 & 50 & 20 & 1.00 & 150 & 500 \\
Class 14 & 50 & 50 & 1.00 & 150 & 500 \\
Class 15 & 150 & 20 & 1.00 & 150 & 500 \\
Class 16 & 150 & 50 & 1.00 & 150 & 500 \\
\hline
\end{tabular}

trucks are prohibitive, so that they will not be used on an optimal solution. Thus, the model is equivalent to the case where we only have to decide the number and types of truck to be used, that is, without the decision whether to use the extra trucks. Consider the following additional data for Model 3: number of trucks $K=2, P_{1}^{\prime} \in[40,80], P_{2}^{\prime}=2 \times P_{1}^{\prime}, c_{1}=50$ and 150 and $c_{2}=1,5 \times c_{1}$.

4.1.1. Computational results: Models 1,2 and 3. In the following, we present some computational results of Models 1, 2 and 3. As the models capture different realities, our main aim in this section is to analyse the results of each model separately. However, for convenience, the models were applied to solve examples of the same classes and for simplicity, their results are presented side by side in Tables 3 and 4. A runtime limit of 180s was arbitrarily chosen for solving each of these examples. Although the models represent tactical decisions problems, this (short) time limit seems to be acceptable in practice as each model may have to be solved many times for supporting managerial decisions (eg, performing different sensitivity analysis of the input parameters, investigating different alternative scenarios and applying rolling time horizons implementations, in which a production-logistics plan is determined for the whole planning horizon but only the decisions in initial time periods are implemented against uncertainties in next periods). Table 3 shows the average results for $16 \times 50=800$ instances in classes 1-16 for Models 1, 2 and 3 . The results were analysed according to:

$C T$

GAP
Computational time (in seconds) of AMPL/ CPLEX.

Value $G a p=|L S-L I| /\left(e^{-10}+|L S|\right)($ calculations made by the optimization package).

For Model 1, note that we could not find a feasible solution for a significant number of instances, and that, for all classes, the optimization package used the maximum resolution time and did not prove the optimality of the solution, although it does gets a small $G A P$ for some classes. It can also be seen that the bigger the size of the problem (represented by the number of items and time periods), the greater the difficulty in obtaining good solutions (see the GAP column). In addition, the increase in transportation costs (classes 9-16) also makes it difficult to solve the problem, probably due to a trade-off between higher setup costs and transportation costs.

For Model 2, it can be seen that the package had less difficulty solving these instances. In classes 1 and 9 , the package proved optimality of the solution found for some instances (note that the average time is lower in these classes). The package found at least one feasible solution for all 800 instances generated. Again, we can see that having a larger number of items and periods, the package has more difficulty in solving the problem (classes 8 and 16). Another observation in 
Table 3 Results obtained by AMPL/CPLEX-computational time and GAP

\begin{tabular}{|c|c|c|c|c|c|c|}
\hline & \multicolumn{2}{|c|}{ Model 1} & \multicolumn{2}{|c|}{ Model 2} & \multicolumn{2}{|c|}{ Model 3} \\
\hline & $C T(s)$ & $G A P(\%)$ & $C T(s)$ & $G A P(\%)$ & $C T(s)$ & $G A P(\%)$ \\
\hline Class G1 & 180.00 & 1.69 & 163.40 & 0.17 & 179.99 & 0.46 \\
\hline Class G2 & 180.00 & 1.91 & 180.00 & 0.59 & 180.00 & 0.69 \\
\hline Class G3 & 180.00 & 1.66 & 180.00 & 0.19 & 180.00 & 0.21 \\
\hline Class G4 & 180.00 & $3.66 *$ & 180.00 & 0.35 & 180.02 & 0.30 \\
\hline Class G5 & 180.00 & 3.83 & 180.00 & 0.70 & 180.00 & 1.73 \\
\hline Class G6 & 180.00 & 6.15 & 180.00 & 1.41 & 180.00 & 1.56 \\
\hline Class G7 & 180.00 & $7.25 * *$ & 180.00 & 0.50 & 180.01 & 1.70 \\
\hline Class G8 & 180.00 & - & 180.00 & 15.51 & 180.03 & $12.53 *$ \\
\hline Class G9 & 180.00 & $2.92 * *$ & 160.87 & 0.29 & 179.99 & 0.89 \\
\hline Class G10 & 180.00 & 3.47 & 180.00 & 1.11 & 179.99 & 1.35 \\
\hline Class G11 & 180.00 & $6.56 * * *$ & 180.00 & 0.36 & 180.00 & 0.43 \\
\hline Class G12 & 180.00 & - & 180.00 & 0.63 & 180.01 & 0.60 \\
\hline Class G13 & 180.00 & 4.34 & 180.00 & 0.92 & 179.99 & 2.25 \\
\hline Class G14 & 180.00 & - & 180.00 & 2.07 & 180.00 & 2.14 \\
\hline Class G15 & 180.00 & - & 180.00 & 0.71 & 180.01 & 2.38 \\
\hline Class G16 & 180.00 & - & 180.00 & 21.01 & 180.03 & $22.98 * * * *$ \\
\hline
\end{tabular}

* Could not find a feasible solution for nine instances, which were removed for the determination of the average.

** Could not find a feasible solution for two instances, which were removed for the determination of the average.

*** Could not find a feasible solution for 48 instances, which were removed for the determination of the average.

**** Could not find a feasible solution for 49 instances, which were removed for the determination of the average.

— Unable to find a feasible solution to 50 instances.

Table 4 Results obtained by AMPL/CPLEX-number of nodes and cutting planes

\begin{tabular}{lccc}
\hline & Model 1 & Model 2 & Model 3 \\
\hline Average number of nodes & 9805.117 & 19177.61 & 26486.07 \\
Average number of cutting planes & 10827.53 & 10366.25 & 10389.13 \\
\hline
\end{tabular}

this table is related to the greater difficulty in solving some instances with high transportation costs.

It can be seen that the package also had more difficulty solving some instances for Model 3, particularly in Classes 8 and 16 (instances with more variables). For all the instances, the package used the time limit and no solution for any sample was shown to be the optimal solution.

We also analysed the average number of nodes of the enumeration tree and cutting planes that the computer package used for each model and, as can be seen in Table 4, this highlights the importance of cutting planes in the solution of these models. In general, the package gets good initial solutions through the generation of cutting planes, with up to $10 \%$ of $G A P$ in the first solution (root node) of the instances in which a feasible solution was found within the time limit.

From the analysis of the results shown, we can conclude that the optimization package is reasonably effective in solving the proposed Models 1, 2 and 3 for production lot sizing problems with transportation cost. However, after the 180-s time limit, the $G A P$ is still relatively high for some classes and, particularly for Model 1, the package had difficulty finding feasible solutions.
We also did some additional computational tests with Model 1, increasing the time limit to 7200 s (Table 5). This resulted in the optimization package using all available time (7200s), without proving the solution optimality. However, the GAP was significantly reduced when compared to the results for the 180-s time limit and, furthermore, with the additional time the optimization package obtained a feasible solution for all classes tested. Note that the average number of nodes in the tables shows that the larger the number of variables, the smaller the number of nodes. Considering that the average computational time for each node is the same, the last column presents the computational time spent after finding the best solution. It is possible to see that in the classes with a smaller number of variables (G1,G5,G9 \& G13), the optimization package spent more time trying to prove the optimality of the solution. Finally, the average number of cutting planes are bigger for classes with a higher number of variables (G4,G8,G12 \& G16).

\subsection{Computational analysis of the integrated lot sizing and bin packing problem (one-dimensional case)}

This section tested the model of the integrated lot sizing with one-dimensional packing problem (Model 4). Initially there is a description of how the data for the experiments were generated and, subsequently, some computational results from the solution of the models by AMPL/CPLEX optimization package are given, now with a 3600-s time limit due to the greater difficulty in solving this model. To generate the data described in Table 6, such as the size of the items and size of trucks, the data described in Beasley (1990) was used. 
Table 5 Results obtained by AMPL/CPLEX-Model 1

\begin{tabular}{|c|c|c|c|c|c|}
\hline & $G A P(\%)$ & $\begin{array}{l}\text { Average number } \\
\text { of cutting planes }\end{array}$ & $\begin{array}{l}\text { Average number } \\
\text { of nodes }\end{array}$ & Best node & $\begin{array}{l}\text { Computational time } \\
\text { (s) after best node }\end{array}$ \\
\hline Class G1 & 0.22 & 3939 & 3153474 & 2528068 & 1467.59 \\
\hline Class G2 & 0.37 & 9797 & 1145678 & 1113116 & 210.32 \\
\hline Class G3 & 0.30 & 11666 & 979216 & 946611 & 246.39 \\
\hline Class G4 & 8.34 & 26737 & 276714 & 276654 & 1.60 \\
\hline Class G5 & 0.01 & 3952 & 691866 & 669197 & 235.91 \\
\hline Class G6 & 0.11 & 9022 & 220378 & 220324 & 1.78 \\
\hline Class G7 & 0.11 & 10802 & 153309 & 153269 & 1.87 \\
\hline Class G8 & 0.35 & 25284 & 57311 & 57254 & 7.17 \\
\hline Class G9 & 0.01 & 3707 & 2241601 & 2142791 & 317.37 \\
\hline Class G10 & 0.02 & 9369 & 691569 & 691481 & 0.92 \\
\hline Class G11 & 0.03 & 10972 & 578271 & 578221 & 0.62 \\
\hline Class G12 & 0.29 & 24944 & 179950 & 179894 & 2.26 \\
\hline Class G13 & 0.02 & 3894 & 470835 & 468779 & 31.43 \\
\hline Class G14 & 0.16 & 8619 & 111784 & 111724 & 3.83 \\
\hline Class G15 & 0.20 & 10741 & 102406 & 102349 & 4.04 \\
\hline Class G16 & 0.60 & 24089 & 31470 & 31374 & 21.89 \\
\hline
\end{tabular}

Table 6 Fixed data for Model 4

\begin{tabular}{lcl}
\hline$N$ & $\{5,10\}$ & The number of types of items in a problem is set at 5 or 10 \\
$T$ & $M=\left[\beta \sum_{i=1}^{n} \sum_{t=1}^{T} \frac{w_{i} d_{i t}}{W}\right]$ & The number of time periods in a problem is set at 5 or 10 \\
$M$ & $(20,100)$ & $\begin{array}{l}\text { The number of trucks is obtained using the expression described in Section } 3.4, \text { with the value of } \\
\beta=1,5\end{array}$ \\
$w_{i}$ & 1000 & The size of the items is randomly generated in the range of values $20-100$ \\
$W$ & 100 & The truck size is fixed at 1000 \\
$s_{i t}$ & 3 & The machine setup cost for the production of the items in the periods is equal to 100 \\
$h_{i t}^{+}$ & $\{100,150,200,400,3000\}$ & The inventory cost per unit per period is set at 3
\end{tabular}

The instances below are based on Beasley (1985), who used an average of 500 items for cutting and packing problems. Thus, we used an average demand of 100 items per period for 5 periods or 50 items per period for instances with 10 periods. Therefore, the values of $n$ and $T$ are smaller than those used in the models of the previous sections.

As in previous models, some were fixed values and others were chosen randomly and uniformly in a range. For example, the size of items $w_{i}$ was chosen randomly between 20 and 100 . The demand $d_{i t}$ was randomly generated so that the sum of all items in all the periods was equal to 500 (50-100 or item). For each demand and size of generated items, five instances were generated, one for each shipping cost. Thus, the classification of classes of these tests is defined as follows: class *.1 with shipping $\operatorname{cost} c=100$, class *.2 with $\operatorname{cost} c=150$, class *.3 with cost $c=200$, class *. 4 with $c=400$ and class *.5 with $c$ $=3000$. The data related to the production capacity were generated as described in Section 4.1 with $\alpha=0.85$.

4.2.1. Computational results: Model 4. The results are shown in Table 7 according to the size of the problem, in other words, four sets of three columns are shown combining the different numbers of types of items and time periods, in the following order: $5 \times 5,5 \times 10,10 \times 5$, and $10 \times 10$. The maximum runtime limit (3600s) was used for all instances. OF is the value of the objective function.

For the $5 \times 5$ instances, it can be seen that the optimization package used the maximum resolution time and, in general, did not prove the optimality of the solutions but got GAPs smaller than $0.23 \%$. Whereas for $5 \times 10$ instances, one can note that increasing the number of periods influenced the solution of the problem. This is shown by the increase in the GAPs for solutions found by the optimization package. Note that, in this case, the GAPs obtained in the solution are higher than $1.2 \%$. The quality of the solution found by the optimization package was not influenced by the transport costs.

When the number of types of items is increased and the time periods remain at $5(10 \times 5$ instances $)$, the optimization package produces lower quality solutions (column GAP) compared to $5 \times 5$ instances. Comparing the quality of the solutions found in the $5 \times 10$ and $10 \times 5$ instances, we note that increasing the number of periods of the planning horizon further complicates the problem, when compared to the increase in the number of items. For the $10 \times 10$ instances, the optimization package found solutions with $G A P \mathrm{~s}$, on average, higher than those found 
Table 7 Results of the tests-Model 4

\begin{tabular}{|c|c|c|c|c|c|c|c|c|}
\hline \multirow[t]{2}{*}{ Classes } & \multicolumn{2}{|c|}{$5 \times 5$} & \multicolumn{2}{|c|}{$5 \times 10$} & \multicolumn{2}{|c|}{$10 \times 5$} & \multicolumn{2}{|c|}{$10 \times 10$} \\
\hline & $O F$ & $G A P(\%)$ & $O F$ & $G A P(\%)$ & $O F$ & $G A P(\%)$ & $O F$ & $G A P(\%)$ \\
\hline 1.1 & 5295 & 0.01 & 9596 & 1.44 & 12188 & 1.07 & 21142 & 3.41 \\
\hline 1.2 & 6745 & 0.02 & 12514 & 1.56 & 15234 & 1.12 & 26738 & 2.14 \\
\hline 1.3 & 8197 & 0.02 & 15434 & 2.06 & 18450 & 1.25 & 32715 & 3.17 \\
\hline 1.4 & 14058 & 0.03 & 26768 & 1.87 & 27590 & 1.63 & 55696 & 3.19 \\
\hline 1.5 & 89395 & 0.04 & 175164 & 2.18 & 168456 & 2.15 & 360058 & 4.46 \\
\hline 2.1 & 6471 & 0.02 & 10981 & 1.84 & 14068 & 0.74 & 23226 & 1.69 \\
\hline 2.2 & 7921 & 0.02 & 13881 & 2.70 & 16918 & 0.94 & 29048 & 2.30 \\
\hline 2.3 & 9371 & 0.02 & 16781 & 2.13 & 19768 & 1.13 & 34869 & 2.57 \\
\hline 2.4 & 15171 & 0.03 & 28381 & 3.15 & 31168 & 1.58 & 58408 & 3.54 \\
\hline 2.5 & 89395 & 0.04 & 176681 & 2.30 & 179429 & 2.09 & 360528 & 3.95 \\
\hline 3.1 & 5713 & 0.01 & 10012 & 1.38 & 13361 & 0.82 & 22028 & 1.33 \\
\hline 3.2 & 7163 & 0.01 & 12896 & 1.22 & 16261 & 1.01 & 27808 & 1.55 \\
\hline 3.3 & 8613 & 0.01 & 15808 & 1.28 & 19161 & 1.14 & 33518 & 1.49 \\
\hline 3.4 & 14413 & 0.01 & 27267 & 1.42 & 30761 & 1.42 & 56631 & 1.85 \\
\hline 3.5 & 91183 & 0.04 & 176095 & 1.79 & 179322 & 2.06 & 356878 & 3.07 \\
\hline 4.1 & 3700 & 0.02 & 7305 & 3.34 & 7000 & 2.77 & 13935 & 2.98 \\
\hline 4.2 & 5237 & 0.02 & 10314 & 3.74 & 9927 & 2.86 & 19835 & 2.80 \\
\hline 4.3 & 6687 & 0.02 & 13260 & 4.07 & 12827 & 2.37 & 25654 & 2.40 \\
\hline 4.4 & 12511 & 0.22 & 24842 & 2.37 & 24490 & 1.94 & 48847 & 1.78 \\
\hline 4.5 & 88081 & 0.23 & 173663 & 1.93 & 172992 & 1.66 & 346921 & 2.01 \\
\hline
\end{tabular}

in the smaller problems. Again, it was not possible to detect any specific behaviour in solving this class of problems in relation to the differences in transportation costs.

\section{Concluding remarks and future work}

In this study, MIP models were given for lot sizing problems with production capacity constraints and distribution decisions based on cargo unitization devices and under restrictions of weight limits, volume or value of cargo. The MIP models were solved using the CPLEX solver's branch-and-cut method and the results show that these models can be used to support integrated decisions in lot sizing and distribution of products in some practical situations.

As mentioned, during the development of this study some companies were visited to identify and evaluate possible reallife applications of the models and solution methods of this study. The application of the models and methods developed in this work in real-world case studies to analyse the performance in practice and the benefits of these models is suggested as an interesting future research, as well as the development of customized solution methods for solving some of the models presented, investigating the specific structures and characteristics of these models. Another interesting line for future work would be to expand on these integrated models considering not only the one-dimensional case (weight, volume or load value), but also two-dimensional and three-dimensional cases, in which various dimensions (eg lengths, widths and heights of the items and of the vehicles) are relevant to the loading problem solution. Regarding the lot sizing problem, a relevant extension includes the possibility of a setup carryover and crossover. The idea is to allow a setup state to be maintained from one period to the next (setup carryover), or to allow that a setup operation can be interrupted at the end of a period and resumed at the beginning of the next period (setup crossover). An updated version of the optimization solver will be used in these future computational tests.

Acknowledgements - The authors thank the two anonymous reviewers for their useful comments and suggestions. This research had the financial support of CNPq and FAPESP (Process number 2010/10133-0, 2014/ 01203-5 and 474782/2013-1).

\section{References}

Alvarez-Valdes R, Parreño F and Tamarif JM (2005). A branch-and-cut algorithm for the pallet loading problem. Computers and Operations Research 32(11): 3007-3029.

Amorim P, Belo-Filho M, Toledo FMB, Almeder C and Almada-Lobo B (2013). Lot sizing versus batching in the production and distribution planning of perishable goods. International Journal of Production Economics 146(1): 208-218.

Anily S and Tzur M (2006). Algorithms for the multi-item multi-vehicles dynamic lot sizing problem. Naval Research Logistics 53(2): 157-169.

Baumol WJ and Vinod HD (1970). An inventory theoretic model of freight transport demand. Management Science 16(7): 413-421.

Beasley JE (1985). An exact two-dimensional non guillotine cutting tree search procedure. Operations Research 33(1): 49-64.

Beasley JE (1990). OR-library: Distributing test problems by electronic mail. Journal of the Operational Research Society 41(11): 1069-1072. 
Bertazzi L and Speranza MG (1999). Models and algorithms for the minimization of inventory and transportation costs: A survey. In: Speranza MG and Staehly P (eds). New Trends in Distribution Logistics. Lecture Notes in Economics and Mathematical Systems Vol. 480. Springer-Verlag: Berlin Heidelberg, pp 137-157.

Beullens P (2014). Revisiting foundations in lot sizing - Connections between Harris, Crowther, Monahan, and Clark. International Journal of Production Economics 155: 68-81.

Birgin EG, Lobato RD and Morabito R (2010). An effective recursive partitioning approach for the packing of identical rectangles in a rectangle. Journal of the Operational Research Society 61(2): 306-320.

Brahimi N, Dauzere-Peres S, Najid NM and Nordi A (2006). Single item lot sizing problems. European Journal of Operational Research 168(1): 1-16.

Coffman EG, Garey MR and Johnson DS (1997). Approximation algorithms for bin packing: A survey. In: Hochbaum DS (ed). Approximation Algorithms for N-Hard Problems. PWS Publishing Company: Boston, MA, pp 46-93.

de Matta R and Miller T (2004). Production and inter-facility transportation scheduling for a process industry. European Journal of Operational Research 158(1): 72-88.

Erengüç SS, Simpson NC and Vakharia AJ (1999). Integrated production/distribution planning in supply chains: An invited review. European Journal of Operational Research 115(2): 219-236.

Fumero F and Vercellis C (1999). Synchronized development of production, inventory, and distribution schedules. Transportation Science 33(3): 330-340.

Glock CH, Grosse EH and Ries JM (2014). The lot sizing problem: A tertiary study. International Journal of Production Economics 155: $39-51$.

Holmbom M and Segerstedt A (2014). Economic order quantities in production: From Harris to economic lot scheduling problems. International Journal of Production Economics 155: 82-90.

Jans R and Degraeve Z (2007). Meta-heuristics for dynamic lot sizing: A review and comparison of solution approaches. European Journal of Operational Research 177(3): 1855-1875.

Jans R and Degraeve Z (2008). Modelling industrial lot sizing problems: A review. International Journal of Production Research 46(6): 1619-1643.

Kaminsky P and Simchi-Levi D (2003). Production and distribution lot sizing in a two stage supply chain. IIE Transactions 35(11): 1065-1075.

Kantorovich LV (1960). Mathematical methods of organizing and planning production. Management Science 6(4): 366-422.

Karimi B, Ghomi S M T F and Wilson JM (2003). The capacitated lot sizing problem: A review of models and algorithms. OMEGA 31(5): 365-378.

Lee WS, Han JH and Cho SJ (2005). A heuristic for a multi-product dynamic lot-sizing and shipping problem. International Journal of Production Economics 98(2): 204-214.

Melo RA and Wolsey LA (2010). Uncapacitated two-level lot-sizing. Operations Research Letters 38(3): 241-245.

Molina F, Morabito R and de Araujo SA (2013). Modelos matemáticos para problemas de dimensionamento de lotes com restrições de capacidade e custos de transporte. Gestão \& Produção 20(3): $573-586$.
Molina F, Santos MO, Toledo FMB and Araujo SA (2009). An approach using Lagrangean/surrogate relaxation for lot-sizing with transportation costs. Pesquisa Operacional 28(2): 269-288.

Morabito R and Pureza V (2009). Loading optimization. In: Raton B (ed) Erdogdu F (org). Optimization in Food Engineering. CRC PressTaylor \& Francis Group: Boca Raton, FL, pp 657-683.

Nascimento MC, Resende MG and Toledo FM (2010). Grasp heuristic with path-relinking for the multiplant capacitated lot sizing problem. European Journal of Operational Research 200(3): 747-754.

Norden L and Velde S (2005). Multi-product lot-sizing a transportation capacity reservation contract. European Journal of Operational Research 165(1): 127-138.

Rizk N and Martel A (2001). Supply chain flow planning methods: A review of the lot-sizing literature. Working Paper DT-2001-AM-1, Université Laval, QC, Canada.

Robinson P, Narayananb A and Sahinc F (2009). Coordinated deterministic dynamic demand lot-sizing problem: A review of models and algorithms. Omega 37(1): 3-15.

Sambasivan M and Schmidt CP (2002). A heuristic procedure for solving multi-plant, multiitem, multi-period capacitated lot-sizing problems. Asia-Pacific Journal of Operational Research 19: 87-105.

Sambasivan M and Yahya S (2005). A Lagrangean-based heuristic for multi-plant, multi-item, multi-period capacitated lot-sizing problems with inter-plant transfers. Computers \& Operations Research 32(3): 537-555.

Sarmiento AM and Nagi N (1999). A review of integrated analysis of production-Distribution systems. IIE Transactions 31(11): 1061-1074.

Seliaman ME and Ahmad AR (2009). A generalized algebraic model for optimizing inventory decisions in a multi-stage complex supply chain. Transportation Research Part E 45(3): 409-418.

Stecke KE and Zhao X (2007). Production and transportation integration for a make-to-order manufacturing company with a commit-todelivery business mode. Manufacturing \& Service Operations Management 9(2): 206-224.

Thomas DJ and Griffin PM (1996). Coordinated supply chain management. European Journal of Operational Research 94(11): 1-15.

Vidal CJ and Goetschalckx M (1997). Strategic production-distribution models: A critical review with emphasis on global supply chain models. European Journal of Operational Research 98(1): 1-18.

Vroblefski M, Ramesh R and Zionts S (2000). Efficient lot-sizing under a differential transportation cost structure for serially distributes warehouses. European Journal of Operational Research 127(3): 574-593.

Wong JT (2010). The distribution processing and replenishment policy of the supply chain under asymmetric information and deterioration: Insight into the information value. Expert Systems with Applications 37: 2347-2353.

Xiao Y and Taaffe K (2010). Satisfying market demands with delivery obligations or delivery charges. Computers \& Operations Research 37(2): 396-405.
Received 14 January 2015; accepted 29 January 2016 after two revisions 